\title{
Die freiwillige „Übererfüllung“ unionsrechtlicher Vorgaben durch die Mitgliedstaaten. \\ Ein Beitrag zur rechtsdogmatischen und rechtspolitischen Diskussion um das sog. „Gold Plating“6
}

\author{
Von Franz Leidenmühler, Linz*
}

Innerhalb der Phänomena mitgliedstaatlicher freiwilliger Übererfüllung von Unionsrecht ist scharf zwischen der autonomen Übernahme unionsrechtlicher Vorgaben außerhalb des Anwendungsbereichs und der Verschärfung des materiellen Regelungsgehalts innerhalb des Anwendungsbereichs der Unionsnorm zu trennen. Letzteres wird zunehmend als „Fleißaufgabe“ des nationalen Gesetzgebers negativ konnotiert. Der vorliegende Beitrag unternimmt eine präzise Begriffsabgrenzung und stellt die Konsequenzen in Hinblick auf Fragen der unionsrechtskonformen Interpretation sowie der Vorlageberechtigung und -verpflichtung dar. Zudem wird ausgeführt, dass ein Verzicht der Mitgliedstaaten auf das sog. ,, Gold Plating " primärrechtlich weder gefordert noch intendiert ist, sondern vielmehr in Widerspruch zu den Grundsätzen der Subsidiarität und der Verhältnismäßigkeit steht.

\section{Einleitung}

„Nach Golde drängt, am Golde hängt doch alles.“ - Schon Margaretes Seufzer in Goethes Faust ${ }^{1}$ deutet an, dass höchsten Ansprüchen genügende und dementsprechend so bezeichnete „Goldstandards" vormalig durchaus als erstrebenswert angesehen wurden. Während vor diesem Hintergrund der Begriff des „Gold Plating“ im wissenschaftlichen Diskurs überwiegend wertfrei gebraucht ${ }^{2}$ oder positiv ${ }^{3}$ belegt wurde und wird, kommt es neuerdings insb. in der rechtspolitischen und politischen Diskussion immer häufiger zu einer negativen Konnotation ${ }^{4}$ als unnötige „Fleißaufgabe“ des nationalen Gesetzgebers bei der Umsetzung unionsrechtlicher Vorgaben. ${ }^{5}$

Ein Vorreiter bei der Zurückdrängung des „Gold Plating“ ist Österreich. So finden sich in den Regierungsprogrammen seit dem Jahr 2000 regelmäßig Bekenntnisse

* Prof. Dr. Franz Leidenmühler ist Vorstand des Instituts für Europarecht der Johannes Kepler Universität Linz/ Österreich.

1 J. W. von Goethe, Faust. Der Tragödie erster Teil, Kapitel 11, 1808.

2 Vgl. nur F. Burmeister/E. Staebe, Grenzen des sog. Gold Plating bei der Umsetzung europäischer Richtlinien in nationales Recht, EuR 2009, S. 444; T. Ambler/F. Chittenden/M. Obodovski, How much Regulation is Gold Plate?, 2004.

3 Vgl. etwa T. Wright/H. Conley, It's better to „gold-plate“ equality law than protect institutional prejudice, The Guardian v. 16.9.2013.

4 Vgl M. Klamert, Altes und Neues zur Harmonisierung im Binnenmarkt, EuZW 2015, S. 265, 267.

5 Zur aktuellen Diskussion um Zweckmäßigkeit und Zulässigkeit von mitgliedstaatlichem „Gold Plating“ siehe eingehend F. Leidenmühler, Entbürokratisierung und Deregulierung im unionsrechtlichen Kontext - Wer hat den Nutzen? (Studie im Auftrag der Arbeiterkammer Wien, 2018). 
zur Vermeidung künftigen und zum Rückbau bestehenden „Gold Platings“, 6 die immer wieder auch Niederschlag in sog. Deregulierungsgesetzen gefunden haben. ${ }^{7}$ Im aktuellen Regierungsprogramm 2017-2022 für die XXVI. GP ${ }^{8}$ wird die Beseitigung des „Gold Plating“ gar als Aufgabe höchster Priorität definiert, der entsprechend breiter Raum eingeräumt wird. ${ }^{9}$

Österreich ist aber keineswegs ein Einzelfall; auch in Deutschland findet sich im Koalitionsvertrag zwischen CDU, CSU und SPD für die 19. Legislaturperiode vom 12.3.2018 das Bekenntnis, europäische Vorgaben nicht mit zusätzlichen bürokratischen Belastungen zu versehen sowie zur ,1:1-Umsetzung von EU-Regelungen “. ${ }^{10}$ Ebenso wird im Noch-immer-Mitgliedstaat ${ }^{11}$ Vereinigtes Königreich ${ }^{12}$ sowie in einigen weiteren Mitgliedstaaten (z.B. NL, DK) ${ }^{13}$ eine Politik des „no Gold Plating“ betrieben. Aber auch seitens der Kommission findet sich im Zuge ihrer Agenda für eine „Bessere Rechtsetzung“14 im Unionsverbund immer wieder die - durch die Arbeiten von Think Tanks begleitete und untermauerte ${ }^{15}$ - Forderung an die Mitgliedstaaten, ,bei der Umsetzung von EU-Rechtsvorschriften in nationales Recht ungerechtfertigtes , Gold plating' zu vermeiden“"16.

6 Zur Historie der Bekämpfung des sog. „Gold Plating“ in der österreichischen legistischen Praxis vgl. eingehend D. Liebwald, Europäische Rechtsbegriffe und österreichische Rechtssprache: Die Transformation von EU-Richtlinien in nationales Recht, JRP 2013, S. 294, 305 ff.

7 Inzwischen wieder aufgehoben: § 1 Abs. 1 DeregulierungsG 2001, öBGB1. I 151/2001; dazu P. Bußjäger, Symbolische Gesetzgebung als Realität und Rechtsproblem: Das Deregulierungsgesetz 2001, ÖJZ 2004, S. 701. Aktuell in Geltung: § 1 Abs. 4 DeregulierungsgrundsätzeG 2017, öBGB1. I 45/2017; dazu H.-P. Leho$\mathrm{fer}$, Das neue Arbeitsprogramm der Bundesregierung: Sunset Boulevard? Die Bundesregierung will - wieder einmal - die Regelungsflut eindämmen, ÖJZ 2017, S. 145.

8 Vgl. ÖVP/FPÖ-Regierungsprogramm 2017-2022 („Kurz“) v. 16.12 .2017 („Zusammen. Für unser Österreich").

9 Gleich an neun Stellen des Programms findet sich das paradigmatische Bekenntnis, die „Übererfüllung von Richtlinien [durch] Gold Plating" zurückzunehmen bzw. in der Zukunft zu unterlassen. Vgl. ÖVP/FPÖ̈-Regierungsprogramm 2017-2022 (,Kurz“) v. 16.12.2017 („Zusammen. Für unser Österreich“) S. 23, 125, 132, $133,134,140,146,156,159$.

10 Vgl. den Koalitionsvertrag zwischen CDU, CSU und SPD für die 19. Legislaturperiode („Ein neuer Aufbruch für Europa - Eine neue Dynamik für Deutschland - Ein neuer Zusammenhalt für unser Land") v. 12.3.2018, S. 43, 64. Dazu sowie zur Vorgeschichte eingehend M. Payrhuber/U. Stelkens, „1:1-Umsetzung“ von EURichtlinien: Rechtspflicht, rationales Politikkonzept oder (wirtschafts)politischer Populismus? - zugleich zu Unterschieden zwischen Rechtsangleichungs- und Deregulierungsrichtlinien, EuR 2019, S. 190.

11 Durch den im Einvernehmen mit dem Vereinigten Königreich gefassten Beschluss (EU) 2019/584 des Europäischen Rates vom 11.4.2019 wurde die Frist nach Art. 50 Abs. 3 EUV bis 31.10.2019 verlängert (ABl. EU 2019 L 101/1).

12 Siehe HM Government, Transposition Guidance - How to implement European Directives effectively, 2018, S. 7 f.: „How do I avoid over-implementation or gold-plating?“. Näher zu UK vgl. E. Pürgy, Better Regulation im Vereinigten Königreich, ZfV 2010, S. 189.

13 Vgl. dazu J. H. Jans/L. Squintani/A. Aragão/R. Macrory/B. W. Wegener, Gold Plating of European Environmental Measures, 2009, S. 4 f., 12 ff., 17.

14 Siehe Europäische Kommission, Mitteilung „Bessere Ergebnisse durch bessere Rechtsetzung - Eine Agenda der EU“, 19.5.2015, KOM (2015) 215 endg. Vgl dazu W. Schroeder, Better Regulation und REFIT - Was bringt die neue EU-Initiative für bessere Rechtsetzung?, wbl. 2016, S. 361; A. Alemanno, How Much Better is Better Regulation?, European Journal of Risk Regulation 2015, S. 344; E. van den Abeele, „Better Regulation": a bureaucratic simplification with a political agenda, 2015; L. Vogel, Bessere Rechtsetzung: eine kritische Betrachtung, 2011.

15 Vgl. insb. Hochrangige Gruppe im Bereich Verwaltungslasten (sog. „Stoiber-Gruppe“), Bürokratieabbau in Europa - Abschlussbericht vom 24.7.2014, S. 9, 52.

16 Vgl. Europäische Kommission, Mitteilung „Bessere Ergebnisse durch bessere Rechtsetzung - Eine Agenda der EU“, 19.5.2015, KOM (2015) 215 endg., S. 8; dahingehend auch schon Europäische Kommission, Pro- 
In vorliegendem Beitrag wird vor dem Hintergrund der im rechtswissenschaftlichen Diskurs um das „Gold Plating“ herrschenden Begriffsverwirrung und -vermengung ${ }^{17}$ eingangs eine definitorische Annäherung sowie eine Abgrenzung zu verwandten Phänomena der freiwilligen „Übererfüllung“ unionsrechtlicher Vorgaben durch die Mitgliedstaaten unternommen. Im Anschluss wird dargestellt, dass ein Verzicht der Mitgliedstaaten auf das sog. „Gold Plating“ vertraglich weder gefordert noch intendiert ist, sondern den dahingehenden Versuchen der Kommission, mitgliedstaatliches „Gold Plating“ zu verpönen, sogar eine Reihe primärrechtlicher Schranken entgegensteht. Abschließend soll dargelegt werden, dass gewichtige rechtspolitische Argumente dafür sprechen, dass die Mitgliedstaaten auch weiterhin von ihren vertraglich eingeräumten Handlungsspielräumen zugunsten der Beibehaltung bzw. Neueinführung höherer nationaler Schutzstandards Gebrauch machen und sich nicht freiwillig dieser wesentlichen Gestaltungsspielräume begeben.

\section{Die verschiedenen Dimensionen der freiwilligen „Übererfüllung“ unionsrechtlicher Vorgaben durch die Mitgliedstaaten}

Eine freiwillige „Übererfüllung“ unionsrechtlicher Vorgaben durch die Mitgliedstaaten ist in unterschiedlichen Dimensionen denkbar, wobei diese im Schrifttum nicht immer sauber auseinandergehalten werden. Vor allem werden die Phänomena der ,,autonomen Übernahme“ unionsrechtlicher Normen (insb. der sog. ,überschießenden Richtlinienumsetzung") und des sog. „Gold Plating“ regelmäßig synonym gebraucht. ${ }^{18}$ Nun ist zwar tatsächlich „Gold Plating“ kein normativer Begriff, sondern ein Kunstbegriff, der in einem sehr unterschiedlichen Sinne verwendet werden kann und wird. ${ }^{19}$ Jedoch bestehen, wie im Folgenden zu zeigen ist, wesentliche strukturelle Unterschiede mit daraus folgenden unterschiedlichen rechtlichen Konsequenzen zwischen einer „Übererfüllung“ unionsrechtlicher Vorgaben inner- und außerhalb des Geltungsbereiches der Unionsrechtsordnung, sodass diese - auch begrifflich - scharf zu trennen sind. Aus diesem Grunde soll

gramm zur Gewährleistung der Effizienz und Leistungsfähigkeit der Rechtsetzung (REFIT): Bestandsaufnahme und Ausblick, 18.6.2014, KOM (2014) 368 endg., S. 7 ff.

17 So auch K. Atthoff/M. Wallgren, Clarifying Gold-Plating - Better Implementation of EU Legislation, Swedish Better Regulation Council, 2013; D. Liebwald (Fn. 6), JRP 2013, S. 294, 305; T. Riehm, Die überschießende Umsetzung vollharmonisierender EG-Richtlinien im Privatrecht, JZ 2006, S. 1035, 1036.

18 Vgl nur W. Schroeder, Der Abbau von „Goldplating“ durch nationale Deregulierung und das Europäische Unionsrecht, ÖGFE Policy Brief 22/2018, S. 5; H. Eberhard/M. Spitzer, Verbraucherschutz und Verfassungsrecht, ÖJZ 2017, S. 308, 310 ff.; J. Stabentheiner, Grundüberlegungen zur Umsetzung der VerbraucherrechteRichtlinie in Österreich, in: Welser (Hrsg.), Die Umsetzung der Verbraucherrechte-Richtlinie in den Staaten Zentral- und Osteuropas, 2015, S. 165, 169 f.; J. Schwarze, Das Verhältnis von nationalem Recht und Europarecht im Wandel der Zeit, Band II, 2013, S. 121 f.; D. Liebwald (Fn. 6), JRP 2013, S. 294, 305; F. Burmeister/E. Staebe (Fn. 2), EuR 2009, S. 444, 445; T. Jäger, Überschießende Richtlinienumsetzung im Privatrecht, 2006, S. 51, 68 ff.; T. Riehm (Fn. 17), JZ 2006, S. 1035, 1045; G. Brandner, Die überschießende Umsetzung von Richtlinien, 2003, S. $12 \mathrm{ff}$.

19 Vgl W. Schroeder, Die Beseitigung von „Gold Plating“ durch nationale Deregulierung und das Unionsrecht, JRP 2018, S. 227, 228. 
nachstehend eine Darstellung der verschiedenen Facetten der mitgliedstaatlichen „Übererfüllung“ erfolgen und eine präzise Begriffsabgrenzung vorgenommen werden. ${ }^{20}$

\section{Autonome Übernahme unionsrechtlicher Vorgaben außerhalb ihres Anwendungsbereiches}

Die Mitgliedstaaten können unionsrechtliche Vorgaben freiwillig über ihren unionsrechtlich vorgegebenen Anwendungsbereich hinaus in das nationale Recht umsetzen oder Unionsrecht außerhalb seines sachlichen, persönlichen oder räumlichen Geltungsbereiches für anwendbar erklären. ${ }^{21}$ Diese fallweise fälschlich ,unechtes Gold Plating“"22, zumeist „überschießende (Richtlinien-)Umsetzung“23, aber auch „erweiterte Rechtsangleichung“"24, „freiwillige Rechtsanpassung“"25, „erweiternde Umsetzung“"26 oder ,autonome Harmonisierung“"27 genannte (bewusste oder unbewusste) Erstreckung des Regelungsgehalts unionsrechtlicher Normen auf Sachverhalte außerhalb ihres Anwendungsbereiches soll im Folgenden als ,autonome Übernahme“ bezeichnet werden.

Im Zuge einer solchen ,autonomen Übernahme“ regelt der nationale Gesetzgeber benachbarte Sachbereiche nach dem Vorbild unionsrechtlicher Normen, die mit der Vorgabe direkt nichts zu tun haben, da sie außerhalb ihres Anwendungsbereichs liegen. ${ }^{28}$ Die Erweiterung kann sich dabei auf den sachlichen, persönlichen, räumlichen, u.U. auch zeitlichen Anwendungsbereich der Unionsnorm beziehen. ${ }^{29}$ Eine , autonome Übernahme“ liegt somit immer dann vor, wenn eine nationale Umsetzungsvorschrift über den Anwendungsbereich der unionsrechtlichen Vorgabe hinausreicht, ${ }^{30}$ sodass es sich im eigentlichen Sinne gar nicht um eine „Umsetzung“ handelt.

20 Dahingehend auch M. Habersack/C. Mayer, Die überschießende Umsetzung von Richtlinien, in: Riesenhuber (Hrsg.), Europäische Methodenlehre, 3. Aufl. 2015, S. 297, 303 m.w.N.

21 Siehe dazu EuGH, Rs. 166/84 (Thomasdünger/Oberfinanzdirektion Frankfurt am Main), ECLI:EU:C:1985:373, Slg. 1985, 3001.

22 Siehe insb. F. Burmeister/E. Staebe (Fn. 2), EuR 2009, S. 444, 445.

23 So erstmals M. Habersack/C. Mayer, Die überschießende Umsetzung von Richtlinien, JZ 1999, S. 913. Vgl. weiter T. Jäger (Fn. 18), S. 25; T. Riehm (Fn. 17), JZ 2006, S. 1035; B. Gsell, Vorlageverfahren und überschieBende Umsetzung von Europarecht, in: Gsell/Hau (Hrsg.), Zivilgerichtsbarkeit und Europäisches Justizsystem, 2012, S. 123; T. Kuhn, Überschießende Umsetzung bei mindest- und vollharmonisierenden Richtlinien: Einheitliche oder gespaltene Anwendung?, EuR 2015, S. 216.

24 Vgl. R. Schulze, Einführung, in: Schulze (Hrsg.), Auslegung europäischen Privatrechts und angeglichenen Rechts, 1999, S. 9, $17 \mathrm{f}$.

25 Vgl. W.-H. Roth, Europäisches Recht und nationales Recht, in: Canaris u.a. (Hrsg.), 50 Jahre Bundesgerichtshof, Bd. II, 2000, S. $847,880 \mathrm{ff}$.

26 Vgl. S. Perner, Erweiternde Umsetzung von Richtlinien des Europäischen Verbraucherrechts, ZfRV 2011, S. 225; so auch schon T. Riehm (Fn. 17), JZ 2006, S. 1035, 1036, 1045.

27 Vgl. Y. Schnorbus, Autonome Harmonisierung in den Mitgliedstaaten durch Inkorporation von Gemeinschaftsrecht, RabelsZ 2001, S. 654.

28 Vgl. F. Burmeister/E. Staebe (Fn. 2), EuR 2009, S. 444, 445.

29 Vgl. T. Jäger (Fn. 18), S. 51 ff.; M. Habersack/C. Mayer (Fn. 20), S. 297, 301 ff.

30 Vgl. M. Habersack/C. Mayer (Fn. 20), S. 297, 298. 
Eine ,autonome Übernahme“ ist auch im Falle von vollharmonisierenden Richtlinien $^{31}$ unionsrechtlich völlig unproblematisch, da es sich ja nicht um eine (diesfalls unzulässige) Standarderhöhung im Anwendungsbereich der Richtlinie handelt, sondern eine Übernahme der Vorgaben der Richtlinie gerade außerhalb ihres Anwendungsbereiches erfolgt. ${ }^{32}$ Ebenso wäre eine freiwillige Rechtsanpassung außerhalb des Geltungsbereiches der unionsrechtlichen Vorgabe im Falle von Verordnungen denkbar.

Die Gründe für die „autonome Übernahme“ unionsrechtlicher Vorgaben durch den mitgliedstaatlichen Gesetzgeber außerhalb ihres Geltungsbereiches liegen vor allem in der Wahrung der Systemeinheit und der Homogenität der nationalen Rechtsordnung sowie in der Vermeidung sachlich nicht rechtfertigbarer Differenzierungen. ${ }^{33}$ So hat beispielsweise die von einigen Mitgliedstaaten regelmäßig vorgenommene „autonome Übernahme“ von verbraucherrechtlichen Richtlinien in die innerstaatliche Zivilrechtsordnung zu einem beträchtlichen Einfluss des europäischen Verbraucherschutzrechts (und nur dafür hat die Union eine Kompetenz) auf das allgemeine Zivilrecht, das weiterhin im autonomen Regelungsbereich der Mitgliedstaaten liegt, geführt (sog ,Spill-over-Effekt“). ${ }^{34}$

\section{Erlass strengerer Schutzmaßnahmen innerhalb des Anwendungsbereiches unionsrechtlicher Vorgaben}

Als gänzlich anders gelagerte „Übererfüllung“ unionsrechtlicher Vorgaben stellt sich dagegen dar, wenn bei der Umsetzung einer unionsrechtlichen Vorgabe in-

31 Während im Falle einer Richtlinie, die das Konzept der Mindestharmonisierung verfolgt, den Mitgliedstaaten eine Anwendung strengerer nationaler Vorschriften schon aufgrund einer richtlinienimmanenten Ermächtigung möglich ist (vgl. etwa Art. 8 Abs. 2 Verbrauchsgüterkauf-Richtlinie [RL 1999/44/EG des EP und des Rates vom 25.5.1999, ABl. EG 1999 L 171/12]), erlaubt das Konzept der abschließenden Harmonisierung bzw. Vollharmonisierung im Geltungsbereich der Richtlinie auch keine strengeren nationalen Vorschriften (vgl. etwa Art. 4 Verbraucherrechte-Richtlinie [RL 2011/83/EU des EP und des Rates vom 25.10.2011, AB1. EU 2011 L 304/64]). Siehe zum Ganzen EuGH, Rs. C-44/01 (Pippig/Hartlauer), ECLI:EU:C:2003:205, Slg. 2003, I-3095, Rn. 40, 44. Zur Terminologie vgl. N. Reich, Von der Minimal- zur Voll- zur Halbharmonisierung, ZEuP 2010, S. 7.

32 S. Perner weist in diesem Zusammenhang zutreffend darauf hin, dass die ,überschießende Umsetzung“ insofern europarechtlich unproblematisch ist, als diesbezüglich weder eine unionsrechtliche Pflicht noch ein unionsrechtliches Verbot besteht und diese vielmehr ein Problem des innerstaatlichen Rechts darstellt. Vgl. $S$. Perner (Fn. 26), ZfRV 2011, S. 225; dahingehend auch M. Habersack/C. Mayer (Fn. 20), S. 297, 306.

33 Zur Frage der (national- bzw. unionsrechtlichen Pflicht zur) richtlinienkonformen Auslegung im Überschussbereich vgl. näher unten, 3.a).

34 Als Beispiel kann die ,autonome Übernahme“ der Verbrauchsgüterkauf-Richtlinie (RL 1999/44/EG des EP und des Rates vom 25.5.1999, AB1. EG 1999 L 171/12) in mitgliedstaatliche Zivilrechtsordnungen dienen. Die Abhilfeinstrumente der Verbraucher bei Vertragswidrigkeit eines Gutes sind nach Art. $1 \mathrm{ff}$. Verbrauchsgüterkauf-Richtlinie nur für Verträge zwischen „Verbrauchern“ und „Verkäufern“ vorzusehen. Da aber ein Gewährleistungs-Sonderregime nur für Verbraucherverträge nicht sinnvoll ist, wurde die Umsetzung in einigen mitgliedstaatlichen Rechtsordnungen nicht auf Verbraucherverträge beschränkt, sondern ist durch Reform des allgemeinen Gewährleistungsrechts erfolgt, wodurch auch Verträge zwischen Unternehmen einbezogen wurden (Ausdehnung des persönlichen Anwendungsbereichs). Vgl. zum Ganzen T. Träger, Zum Systemdenken im europäischen Schuldvertragsrecht - Probleme der Rechtsangleichung durch Richtlinien am Beispiel der Verbrauchsgüterkauf-Richtlinie, ZEuP 2003, S. 525; R. Welser, Das neue Gewährleistungsrecht, ecolex 2001, S. 420 . 
nerhalb des Anwendungsbereiches höhere Standards eingezogen werden, als vom betreffenden Unionsakt - i.d.R. wird es sich um die Rechtssatzform der Richtlinie handeln - zwingend gefordert. Diese Verschärfung des materiellen Regelungsgehalts innerhalb des Anwendungsbereichs aus Anlass der Umsetzung eines Unionsrechtsaktes wird fallweise als ,modifizierende Umsetzung “35, häufiger als ,,inhaltliche Übererfüllung“36 oder (echtes ${ }^{37}$ ) „Gold Plating“, bezeichnet. Dabei geht der nationale Gesetzgeber über den von der Richtlinie vorgegebenen Regelungsauftrag hinaus, ohne den Anwendungsbereich des nationalen Rechts gegenüber der Richtlinie zu erweitern. Von der ,autonomen Übernahme“ unterscheidet sich diese inhaltliche Übererfüllung insofern, als das die unionsrechtliche Vorgabe „übererfüllende“" nationale Recht innerhalb des Anwendungsbereiches der Unionsnorm liegt. 38

Dabei kann eine solche modifizierende Umsetzung hinsichtlich des Regelungsinhaltes sowohl die Tatbestandsvoraussetzungen als auch die Rechtsfolgenseite der unionsrechtlichen Vorgabe betreffen. ${ }^{39}$ Beispiele wären die Ausschöpfung des von einem Unionsrechtsakt eingeräumten Spielraums bis zur festgelegten Obergrenze; die Auswahl der strengsten Variante im Falle mehrerer Umsetzungsoptionen; die Hinzufügung von regulatorischen Anforderungen, die über das im Unionsrechtsakt Geforderte hinausgehen; Ausnahmeregelungen im Unionsrechtsakt, die einem Mitgliedstaat erlauben, lockerere Regelungen zu implementieren, werden nicht in Anspruch genommen; die Umsetzung einer Richtlinie wird dafür genutzt, dass nationale regulatorische Anforderungen eingeführt werden, die nicht im Zusammenhang mit dem Ziel der Richtlinie stehen; die Einführung strengerer Sanktionen oder anderer strengerer Durchsetzungsmechanismen als unionsrechtlich gefordert.

Jedenfalls wird nach der herrschenden Begriffsvorstellung unter sog. „Gold Plating" nur eine inhaltliche Übererfüllung eines unionsrechtlichen Regelungsauftrages durch den mitgliedstaatlichen Gesetzgeber durch Neuerlass entsprechender strengerer Umsetzungsregelungen verstanden. Diese Regelungen müssen zudem aus Anlass eines Unionsrechtsakts erlassen werden, was bedeutet, dass der innerstaatliche Rechtsakt in einem kausalen und auch einem gewissen zeitlichen $\mathrm{Zu}$ sammenhang mit dem Unionsrechtsakt stehen muss. Damit ist vom Begriff des sog. „Gold Plating“ nur die aktive Erlassung nationaler Rechtsvorschriften aus Anlass eines Unionsrechtsaktes, mit der über die Vorgaben dieses Aktes hinaus

35 In Abgrenzung zur ,erweiternden Umsetzung“ (siehe oben 1.). Vgl. S. Perner (Fn. 26), ZfRV 2011, S. 225; T. Riehm (Fn. 17), JZ 2006, S. 1035, 1036, 1045.

36 Vgl. M. Habersack/C. Mayer (Fn. 20), S. 297, 303; dahingehend auch die Definition des österreichischen Bundeskanzleramts: „Schaffung strengerer Regelungen bei Umsetzung einer EG-Richtlinie als von der Richtlinie gefordert" (Rundschreiben BKA-VD, GZ 600.824/011-V/2/01, 2001). Dieselbe Definition verwendet das österreichische Bundeskanzleramt im Österreichischen Handbuch „Bessere Rechtsetzung“ (September 2008).

37 Dies von jenen Autoren, welche das Phänomenon der ,autonomen Übernahme“ unpassenderweise als ,unechtes Gold Plating“ bezeichnen wollen. Siehe insb. F. Burmeister/E. Staebe (Fn. 2), EuR 2009, S. 444, 445.

38 Vgl. M. Habersack/C. Mayer (Fn. 20), S. 297, 303.

39 Vgl. T. Jäger (Fn. 18), S. 52 f. 
zusätzliche Anforderungen, Verpflichtungen oder Standards für die Rechtsunterworfenen eingeführt werden, erfasst. Die bloße Beibehaltung in der Rechtsordnung bereits bestehender strengerer Regeln kann damit nicht als „Gold Plating“ bezeichnet werden. ${ }^{40}$ Ebenso wäre daher auch für die ,zufällige“ Existenz höherer nationaler Schutzstandards, die nicht in Zusammenhang mit der Erlassung nationaler Rechtsvorschriften aus Anlass eines Unionsrechtsaktes stehen, die Verwendung des Begriffs des „Gold Plating“ unzutreffend.

Damit kann „Gold Plating“ als Übererfüllung unionsrechtlicher Vorgaben durch die Erlassung nationaler Rechtsvorschriften aus Anlass eines Unionsrechtsaktes, mit der über die Vorgaben des Unionsrechtsaktes hinaus im Anwendungsbereich des Unionsrechts zusätzliche Anforderungen, Verpflichtungen oder Standards für die Rechtsunterworfenen eingeführt werden, definiert werden.

Eine vergleichbare Umschreibung findet sich auch in der Definition durch das österreichische Justizministerium. ${ }^{41}$ Trotz der hier auf Zustimmung treffenden korrekten Definition sind dann aber nicht alle in der Folge aufgeführten Beispiele für „Gold Plating“ auch passend: So ist etwa die dort genannte Ausdehnung des Anwendungsbereiches über den in der Unionsnorm vorgesehenen Geltungsbereich hinaus gerade kein Fall des „Gold Plating“, sondern einer der ,autonomen Übernahme“" (siehe oben 1.). Und die Umsetzung einer Richtlinie vor Ablauf der Umsetzungsfrist, welche als weiteres Beispiel für „Gold Plating“ genannt wird, ${ }^{42}$ ist wiederum weder als ,autonome Übernahme“ unionsrechtlicher Vorgaben noch als „Gold Plating“ einzuordnen. Da sich nach st. Rsp. des EuGH die verbindliche Wirkung der Ziele einer Richtlinie bereits mit ihrem Inkrafttreten entfaltet und Richtlinien in der Folge auch schon vor Ablauf der Umsetzungsfrist gewisse Vorwirkungen zeitigen (insb. ein Vereitelungsverbot), kann eine Umsetzung durch einen Mitgliedstaat vor Ablauf der Umsetzungsfrist nicht als sog. „Gold Plating“ oder ,überschießende Umsetzung“ angesehen werden, da auch zwischen Inkrafttreten und Fristablauf keine inhaltliche Übererfüllung bestanden hat. ${ }^{43}$

40 Dies wird lediglich vereinzelt als sog. „passives Gold Plating“ benannt. Vgl. M. Pošvanc/T. Púchly/M. Reguli/E. Maurice, The Gold-plating identification problems in Slovakia and Lithuania and possible solutions for EU member states, Policy Paper, 2015, S. 31.

41 Vgl. BMVRDJ-VD, Hinweise für die Verwendung des Arbeitsbehelfs - Gold Plating v. 8.3.2018, GZ: BMVRDJ-601.121/0023-V 2/2018.

42 Vgl. BMVRDJ-VD, Hinweise für die Verwendung des Arbeitsbehelfs - Gold Plating v. 8.3.2018, GZ: BMVRDJ-601.121/0023-V 2/2018. Auch nach einer Definition der Britischen Regierung in ihren „Guiding Principles for EU Legislation“ liegt sog. „Gold Plating“ immer dann vor, wenn die Umsetzung einer unionsrechtlichen Vorgabe (speziell von Richtlinien) über das hinausgeht, was im betreffenden Rechtsakt gefordert wird, mithin auch dann, wenn eine Richtlinie früher als verlangt umgesetzt wird. Vgl. HM Government, Transposition Guidance - How to implement European Directives effectively, 2018, S. 8.

43 Vgl. insb. EuGH, Rs. C-129/96 (Inter-Environnement Wallonie), ECLI:EU:C:1997:628, Slg. 1997, I-7411, Rn. $40 \mathrm{ff}$. 


\section{Rechtsfolgen aus der Abgrenzung}

Während die Begriffsbildung oftmals eine Frage der Zweckmäßigkeit darstellt, ist im gegebenen Falle der Übererfüllung unionsrechtlicher Vorgaben die Abgrenzung zwischen der autonomen Übernahme außerhalb des Anwendungsbereiches des Unionsrechts einerseits und der verschärfenden Umsetzung innerhalb des Anwendungsbereiches andererseits auch von rechtlicher Bedeutung.

\section{a) Verpflichtung zur unionsrechtskonformen Interpretation}

Im Falle von „Gold Plating“ innerhalb des Anwendungsbereiches des Unionsrechts ist das über das unionsrechtlich vorgesehene Niveau hinausgehende nationale Recht jedenfalls im Einklang mit dem Sekundärrecht, das es durchführt, auszulegen, auch wenn es den darin vorgesehenen zwingend vorgeschriebenen Schutzstandard verschärft. ${ }^{44}$

Dagegen ist bei der autonomen Übernahme unionsrechtlicher Vorgaben außerhalb ihres Anwendungsbereiches überaus fraglich, ob die betreffenden nationalen Normen auch im überschießenden Bereich unionsrechts-, d.h. zumeist richtlinienkonform auszulegen sind. ${ }^{45}$ Nach herrschender und zutreffender Auffassung ergibt sich eine solche Pflicht aus dem Unionsrecht nicht. Das unionale Gebot der unionsrechtskonformen Interpretation ${ }^{46}$ beschränkt sich vielmehr auf den Anwendungsbereich der Unionsrechtsordnung. ${ }^{47}$ Auch aus der Rsp. des EuGH lässt sich nichts Gegenteiliges entnehmen. Das vom Gerichtshof regelmäßig formulierte „klare Interesse“ der Union daran, dass die aus dem Unionsrecht übernommenen Bestimmungen oder Begriffe ,unabhängig davon, unter welchen Voraussetzungen sie angewandt werden sollen, einheitlich ausgelegt werden, um künftige Auslegungsunterschiede zu verhindern“48, dient in den betreffenden Urteilen immer dem Zweck, die Zuständigkeit des EuGH im Vorabentscheidungsverfahren zu be-

44 Vgl. W. Schroeder (Fn. 18), S. 3; ders. (Fn. 19), JRP 2018, S. 227, 229.

45 Vgl. M. Habersack/C. Mayer (Fn. 20), S. 297, 307 ff.; J. Koch, Die Einheit der nationalen Rechtsordnung und die europäische Privatrechtsangleichung, JZ 2006, S. 277, 279.

46 Siehe dazu instruktiv A. Hatje, Gemeinschaftskonforme Auslegung des nationalen Rechts - unter besonderer Berücksichtigung des Umweltrechts, in: Rengeling (Hrsg.), Handbuch des europäischen und deutschen Umweltrechts, 2. Aufl. 2003, §33.

47 Vgl. M. Ruffert, in: Calliess/Ruffert (Hrsg.), EUV/AEUV, 5. Aufl. 2016, Art. 288 AEUV, Rn. 83 m.w.N.; M. Nettesheim, in: Grabitz/Hilf/Nettesheim, Das Recht der Europäischen Union, 62. EL. 2017, Art. 288 AEUV, Rn. 131; S. Perner (Fn. 26), ZfRV 2011, S. 225, 228 f.; T. Kuhn (Fn. 23), EuR 2015, S. 216, 217 m.w.N.; M. Habersack/C. Mayer (Fn. 20), S. 297, 309 ff. m.w.N.; D. A. Verse, in: Habersack/Wicke (Hrsg.), BeckOGK zum Umwandlungsgesetz, UmwG § 123, Rn. 16.1.; C. Ohler, Objektive Wirkung von Richtlinien, in: Hummer (Hrsg.), Neueste Entwicklungen im Zusammenspiel von Europarecht und nationalem Recht der Mitgliedstaaten, 2010, S. 147, 158 f.; C. Mayer/J. Schürnbrand, Einheitlich oder gespalten? - Zur Auslegung nationalen Rechts bei überschießender Umsetzung von Richtlinien, JZ 2004, S. 545, 549; C. Herrmann, Richtlinienumsetzung durch die Rechtsprechung, 2003, S. 127.

48 EuGH, Rs. C-28/95 (Leur-Bloem), ECLI:EU:C:1997:369, Slg. 1997, I-4161, Rn. 32; siehe weiters EuGH, verb. Rs. C-297/88 und C-197/89 (Dzodzi), ECLI:EU:C:1990:360, Slg. 1990, I-3763, Rn. 37; EuGH, Rs. C-32/11 (Allianz Hungária), ECLI:EU:C:2013:160, Rn. 21 f. 
gründen. ${ }^{49}$ Daraus kann aber nicht abgeleitet werden, dass die Mitgliedstaaten verpflichtet wären, innerstaatliche Normen auch außerhalb des Anwendungsbereiches des Unionsrechts unionsrechtskonform auszulegen. Ganz im Gegenteil weist der EuGH darauf hin, dass „,ür die Berücksichtigung der Grenzen, die der nationale Gesetzgeber der Anwendung des [Unions]rechts auf rein innerstaatliche Sachverhalte setzen wollte, [...] das nationale Recht [gilt] ${ }^{\text {"50 }}{ }^{51} \mathrm{Im}$ Urteil in der Rs. C-264/96 stellt der EuGH schließlich explizit klar, dass dann, wenn „der Rechtsstreit vor dem nationalen Gericht [...] einen Sachverhalt [betrifft], der nicht in den Anwendungsbereich des Gemeinschaftsrechts fällt, [...] dieses Gericht nach dem Gemeinschaftsrecht weder verpflichtet [ist], seine Rechtsvorschriften gemeinschaftsrechtskonform auszulegen, noch, sie unangewendet zu lassen"52. Ob also im Falle der autonomen Übernahme einer unionsrechtlichen Vorschrift der überschießende Bereich unionsrechtskonform auszulegen ist, ist somit alleine nach nationalem Recht zu entscheiden. ${ }^{53}$ In der Regel wird der nationale Gesetzgeber die Einheitlichkeit der Auslegung und Anwendung einer Norm gewollt haben, sodass dies als systematisches Argument für eine einheitliche (mithin unionsrechtskonforme) und gegen eine ,gespaltene“ Interpretation spricht. ${ }^{54}$

\section{b) Vorlageberechtigung und Vorlageverpflichtung}

Im Falle der Übererfüllung unionsrechtlicher Vorgaben innerhalb des Anwendungsbereiches des Unionsrechts (,Gold Plating“) gelten die allgemeinen Regeln des Art. 267 AEUV, wonach alle Gerichte der Mitgliedstaaten Fragen zur Vorabentscheidung durch den EuGH stellen dürfen und Gerichte, deren Entscheidungen nicht mehr mit Rechtsmitteln des innerstaatlichen Rechts angefochten werden können, dies unter den dort angeführten Voraussetzungen tun müssen.

Auch bei Fragen zur Auslegung von Normen des Primär- oder Sekundärrechts bzw. zur Gültigkeit von Sekundärrecht, die sich einem mitgliedstaatlichen Gericht im überschießenden Bereich im Falle einer autonomen Übernahme stellen, nimmt der Gerichtshof in st. Rsp. eine Befugnis nationaler Gerichte zur Vorlage an. Nationale Gerichte dürfen mithin Vorlagefragen aus dem überschießenden Bereich an den EuGH herantragen, obwohl deren Beantwortung - aus Sicht des Unionsrechts - gar nicht von Entscheidungserheblichkeit ist und diese somit als „hypo-

49 Vgl. D. A. Verse (Fn. 47), UmwG § 123, Rn. 16.1.; S. Perner (Fn. 26), ZfRV 2011, S. 225, 228 f.; a. A. dagegen $W .-H$. Roth (Fn. 25), S. 847, 883 ff.

50 EuGH, Rs. C-28/95 (Leur-Bloem), ECLI:EU:C:1997:369, Slg. 1997, I-4161, Rn. 33; ebenso EuGH, Rs. C-3/04 (Poseidon Chartering), ECLI:EU:C:2006:176, Slg. 2006, I-2505, Rn. 17, wo ausdrücklich auf den Willen des innerstaatlichen Gesetzgebers abgestellt wird.

51 Vgl. D. A. Verse (Fn. 47), UmwG § 123, Rn. 16.1.

52 EuGH, Rs. C-264/96 (Imperial Chemical Industries), ECLI:EU:C:1998:370, Slg. 1998, I-4695, Rn. 34.

53 Vgl. EuGH, Rs. C-3/04 (Poseidon Chartering), ECLI:EU:C:2006:176, Slg. 2006, I-2505, Rn. 17; zum ganzen D. A. Verse (Fn. 47), UmwG § 123, Rn. 16; T. Kuhn (Fn. 23), EuR 2015, S. $216,225$.

54 Vgl. T. Kuhn (Fn. 23), EuR 2015, S. 216, 225, 236; C. Mayer/J. Schürnbrand (Fn. 47), JZ 2004, S. 545, 551; M. Habersack/C. Mayer (Fn. 20), S. 297, 317. 
thetische Fragen“ zu qualifizieren wären. Als Begründung der Zulässigkeit dient dem EuGH, wie schon oben angedeutet, dass diese Vorgangsweise der Rechtssicherheit und der Vermeidung von Unklarheiten dient. ${ }^{55}$ Zuletzt hat der EuGH seine ehemals sehr offene Haltung gegenüber Vorlagen aus reinen Inlandssachverhalten (im Sinne einer ,Vermutung der Entscheidungserheblichkeit ${ }^{656}$ ) aber etwas relativiert und knüpft die Zulässigkeit nun an bestimmte Konstellationen, deren Vorliegen das vorlegende Gericht darzulegen und zu begründen hat. ${ }^{57} \mathrm{Da}$ jedoch explizit jene Situationen, ,in denen zwar der Sachverhalt des Ausgangsverfahrens nicht unmittelbar in den Geltungsbereich des Unionsrechts fällt, aber die Vorschriften des Unionsrechts durch das nationale Recht, das sich zur Regelung rein innerstaatlicher Sachverhalte, deren Merkmale sämtlich nicht über die Grenzen eines Mitgliedstaats hinausweisen, nach den im Unionsrecht getroffenen Regelungen richtete, für anwendbar erklärt wurden“, vom EuGH weiterhin als Grund für die Annahme einer Vorlagefrage aus dem überschießenden Bereich akzeptiert werden, ${ }^{58}$ wird in den hier interessierenden Fällen die Vorlageberechtigung im Falle autonomer Übernahmen kaum tangiert.

Eine ganz andere Frage ist aber, ob im überschießenden Bereich auch eine Verpflichtung letztinstanzlicher Gerichte zur Vorlage besteht, wenn die Auslegung der Unionsnorm entscheidungserheblich ist. Der Gerichtshof hat darüber noch nicht explizit entschieden. Im Schrifttum gehen die Meinungen darüber auseinander. ${ }^{59}$ Fallweise wird aus der Rsp. des EuGH zur Vorlagebefugnis „konsequenterweise" auch eine Vorlagepflicht abgeleitet. ${ }^{60}$ Dieser Zugang verkennt aber, dass die Annahme von freiwilligen Vorlagen innerstaatlicher Gerichte vom EuGH als Hilfestellung verstanden wird, die aus dem wechselseitigen Kooperationsverhältnis abgeleitet wird. ${ }^{61}$ Aufgrund dieser Kooperationsfunktion ist es nachvollziehbar, dass der EuGH auch Fragen zur Auslegung des Unionsrechts beantwortet, die mangels unionsrechtlich gebotener Anwendbarkeit desselben nur aus nationaler Sicht von Entscheidungserheblichkeit für den innerstaatlichen Rechtsstreit sind, nicht jedoch aus Sicht des Unionsrechts. Liegt dem Vorabentscheidungsersuchen vordergründig dieser Zweck der Rechtshilfe zugrunde, weil das Unionsrecht in diesem Fall nicht selbst nach seiner einheitlichen Anwendung verlangt, so kann sich aus unionsrechtlicher Sicht lediglich eine Vorlageberechtigung, nicht jedoch

55 Vgl. EuGH, Rs. C-28/95 (Leur-Bloem), ECLI:EU:C:1997:369, Slg. 1997, I-4161, Rn. 34.

56 Vgl. EuGH, Rs. C-399/11 (Melloni), ECLI:EU:C:2013:107, Rn. 29.

57 Vgl. EuGH, Rs. C-268/15 (Ullens de Schooten), ECLI:EU:C:2016:874, Rn. 50 ff.

58 Vgl. EuGH, Rs. C-268/15 (Ullens de Schooten), ECLI:EU:C:2016:874, Rn. 53.

59 Befürwortend z.B. T. Jäger (Fn. 18), S. 195 ff., 223 ff.; ablehnend dagegen etwa M. Habersack/C. Mayer (Fn. 23), JZ 1999, S. $913,919 \mathrm{ff}$.

60 Vgl. D. A. Verse (Fn. 47), UmwG § 123, Rn. 18; dahingehend auch S. Perner (Fn. 266), ZfRV 2011, S. 225, 230.

61 Vgl. M. Mayrhofer, Das Kooperationsverhältnis im staatlichen Unionsverfassungsrecht, in: Holoubek/Martin/ Schwarzer (Hrsg.), Die Zukunft der Verfassung - Die Verfassung der Zukunft? - Festschrift für Karl Korinek, 2010, S. 373, 396 f.; M. Schmid, Die Grenzen der Auslegungskompetenz des EuGH im Vorabentscheidungsverfahren nach Art. 234 EG, 2005, S. 161; B. Hess, Rechtsfragen des Vorabentscheidungsverfahrens, RabelsZ 66 (2002), S. 471, 486. 
eine Vorlagepflicht (letztinstanzlicher Gerichte) ergeben. ${ }^{62}$ Die Annahme einer Verpflichtung zur Vorlage aus reinen Inlandsachverhalten würde zudem das durch das Prinzip der begrenzten Einzelermächtigung geprägte Kompetenzgefüge zwischen Union und Mitgliedstaaten massiv tangieren und dem vom EuGH formulierten Grundsatz widerstreiten, wonach in Sachverhalten außerhalb des Anwendungsbereiches der Unionsrechtsordnung innerstaatliche Gerichte nach dem Unionsrecht weder verpflichtet sind, ihre Rechtsvorschriften unionsrechtskonform auszulegen, noch, sie unangewendet zu lassen. ${ }^{63}$

\section{III. „Gold Plating“: eine rechtliche Würdigung}

\section{Die unionsrechtliche Zulässigkeit von „Gold Plating“6}

Da jede nicht-minimalistische Umsetzung bzw. Durchführung von Unionsrecht in einem Mitgliedstaat zu nationalen Unterschieden und damit zur Fragmentierung des Binnenmarktes führt, könnte dies dem Zweck der Rechtsangleichung, nämlich durch den Abbau rechtlicher Unterschiede zwischen den Mitgliedstaaten Wettbewerbsverzerrungen im Binnenmarkt zu beseitigen, ${ }^{64}$ zuwiderlaufen. ${ }^{65}$ Jedoch eröffnet die Harmonisierungspolitik der Union den Mitgliedstaaten regelmäBig bewusst einen gewissen Handlungsspielraum, der auch in unterschiedlicher Weise genutzt werden kann. ${ }^{66}$ Daher ist eine Beibehaltung bzw. Neueinführung höherer Standards als vom umsetzungsbedürftigen Sekundärrechtsakt gefordert jedenfalls dort zulässig, wo durch einen Mitgliedstaat ein von einem Sekundärrechtsakt eingeräumter Spielraum bis zur festgelegten Obergrenze ausgeschöpft wird. In diesem Fall ist die gewählte mitgliedstaatliche Umsetzung schon sekundärrechtlich zugelassen und braucht - anders als im Falle einer Mindestharmonisierung - auch nicht an den vertraglichen Vorgaben, insb. an den Grundfreiheiten des Binnenmarktes, gemessen zu werden.

Weiters ist sog. „Gold Plating“ typischerweise im Falle von Richtlinien, die das Konzept der Mindestharmonisierung ${ }^{67}$ verfolgen, denkbar. Auch in diesem Falle wird schon richtlinienimmanent der Weg zu einem höheren nationalen Standard eröffnet, ${ }^{68}$ der lediglich mit dem Vertrag in Einklang zu stehen hat, also insb. mit den Grundfreiheiten kompatibel sein muss. ${ }^{69}$ Sog. ,Gold Plating“ kann aber selbst

$62 \mathrm{Vgl} J$. Eder, „Nützliche Hilfestellung“ oder „Gutachten zu hypothetischen Fragen“? Zur Zulässigkeit von Vorabentscheidungsanträgen bei reinen Inlandssachverhalten, JB1. 2013, S. 403, 405.

63 Vgl. EuGH, Rs. C-264/96 (Imperial Chemical Industries), ECLI:EU:C:1998:370, Slg. 1998, I-4695, Rn. 34.

64 Vgl. EuGH, Rs. 193/80 (Kommission/Italien), ECLI:EU:C:1981:298, Slg. 1981，3019, Rn. 17; EuGH, Rs. C-58/08 (Vodafone), ECLI:EU:C:2010:321, Slg. 2010, I-4999, Rn. $32 \mathrm{ff.}$

65 Vgl. M. Klamert (Fn. 4), EuZW 2015, S. 265, 267; F. Burmeister/E. Staebe (Fn. 2), EuR 2009, S. 444, 455; M. Payrhuber/U. Stelkens (Fn. 10), S. 209.

66 Vgl. W. Schroeder (Fn. 19), JRP 2018, S. 227, 230.

67 Dazu M. Dougan, Minimum Harmonization and the Internal Market, CMLRev. 2000, S. 853, 879.

68 Ebenso W. Schroeder (Fn. 18), S. 4.

69 Vgl. F. Leidenmühler in: Jaeger/Stoeger (Hrsg.), EUV/AEUV, 2018, Art. 114 AEUV, Rn. 24. 
bei vollharmonisierenden Richtlinien erfolgen, ${ }^{70}$ in diesem Fall muss aber, sofern zum jeweiligen Kompetenztatbestand verfügbar, auf eine primärrechtliche Schutzverstärkungsklausel zurückgegriffen werden. ${ }^{71}$

Selbst im Falle von Verordnungen, die an sich ja von den Mitgliedstaaten gar nicht umzusetzendes unmittelbar anwendbares Einheitsrecht schaffen, ist sog. „Gold Plating“ durch höhere nationale Standards dann ausnahmsweise denkbar, wenn Verordnungsbestimmungen den Mitgliedstaaten Durchführungsakte mit Umsetzungsspielraum auftragen. ${ }^{72}$

\section{Die unionsrechtliche Garantie von „Gold Plating“6}

Mitgliedstaatliches sog. „Gold Plating“ ist unter den eben dargestellten Voraussetzungen nicht nur zulässig, sondern entgegen gelegentlich geäußerter Auffassung ${ }^{73}$ sogar primärrechtlich garantiert. Versuchen der Kommission ${ }^{74}$ oder anderer Stakeholder auf Unionsebene ${ }^{75}$, mitgliedstaatliche Standarderhöhungen zu verpönen, steht eine ganze Reihe von vertraglichen Schranken entgegen.

\section{a) Gleichrangigkeit der Ziele der Verträge}

Art. 3 Abs. 3 EUV weist darauf hin, dass die Union auf ,eine in hohem Maße wettbewerbsfähige soziale Marktwirtschaft, die auf Vollbeschäftigung und sozialen Fortschritt abzielt, sowie ein hohes Maß an Umweltschutz und Verbesserung der Umweltqualität hin[wirkt]". Damit bringt diese grundlegende Zielbestimmung klar zum Ausdruck, dass die Union neben der Wettbewerbsfähigkeit von Unternehmen gleichberechtigt auch andere Ziele zu verfolgen hat, was auch von der Kommission eingeräumt wird. ${ }^{76}$ Damit stehen schon die Ziele der Verträge einer generellen Ablehnung mitgliedstaatlicher Abweichungen zugunsten höherer

70 Wie hier W. Schroeder (Fn. 18), S. 4; a. A. T. Riehm (Fn. 17), JZ 2006, S. 1035, 1045; F. Burmeister/E. Staebe (Fn. 2), EuR 2009, S. 444, 449 ff., 453 ff. - Zulässigkeit von „Gold Plating“ nur bei ausdrücklichen „Öffnungsklauseln“; ebenso M. Habersack/C. Mayer (Fn. 2020), S. 297, 306, wonach „eine Vollharmonisierung [...] weitergehendes nationales Recht ausschließt".

71 Siehe etwa Art. 114 Abs. 4 ff. AEUV für die Binnenmarktharmonisierung, Art. 169 Abs. 4 AEUV im Bereich Verbraucherschutz oder Art. 193 AEUV im Bereich Umweltschutz.

72 Als Beispiel hierfür kann die Datenschutz-Grundverordnung (VO [EU] 2016/679 des EP und des Rates vom 27.4.2016, ABl. EU 2016 L 119/1) herangezogen werden, die dieserart eigentlich als Hybridakt zwischen Verordnung und Richtlinie erscheint. Seitens Österreichs wurde im Übrigen im Zusammenhang mit der Umsetzung der DSGVO durch das Datenschutz-Deregulierungsgesetz 2018 (öBGB1. I 24/2018) „Gold Plating“ betrieben.

73 Vgl. etwa F. Burmeister/E. Staebe (Fn. 2), EuR 2009, S. 444, 453 ff.

74 Vgl. Europäische Kommission, Mitteilung „Bessere Ergebnisse durch bessere Rechtsetzung - Eine Agenda der EU“, 19.5.2015, KOM (2015) 215 endg., S. 8; dahingehend auch schon Europäische Kommission, Programm zur Gewährleistung der Effizienz und Leistungsfähigkeit der Rechtsetzung (REFIT): Bestandsaufnahme und Ausblick, 18.6.2014, KOM (2014) 368 endg., S. 7 ff.

75 Vgl. insb. Hochrangige Gruppe im Bereich Verwaltungslasten ( $\operatorname{sog}$ „Stoiber-Gruppe“), Bürokratieabbau in Europa - Abschlussbericht v. 24.7.2014, S. 9, 52.

76 Vgl. Europäische Kommission, Programm zur Gewährleistung der Effizienz und Leistungsfähigkeit der Rechtsetzung (REFIT): Bestandsaufnahme und Ausblick, 18.6.2014, KOM (2014) 368 endg., S. 18 ff. 
Schutzstandards im Sinne einer Verpflichtung zum „copy-and-paste“ von Harmonisierungs-Richtlinien entgegen.

\section{b) Grundprinzipien der Subsidiarität und Verhältnismäßigkeit}

Nach dem in Art. 5 Abs. 3 EUV verankerten Subsidiaritätsprinzip „,wird die Union in den Bereichen, die nicht in ihre ausschließliche Zuständigkeit fallen, nur tätig, sofern und soweit die Ziele der in Betracht gezogenen Maßnahmen von den Mitgliedstaaten weder auf zentraler noch auf regionaler oder lokaler Ebene ausreichend verwirklicht werden können, sondern vielmehr wegen ihres Umfangs oder ihrer Wirkungen auf Unionsebene besser zu verwirklichen sind“. Demnach ist die einzelstaatliche Ebene oftmals sicherlich besser geeignet, das ihr passende Schutzniveau in gesellschaftlich relevanten Bereichen zu definieren. Eine Rechtsangleichung ausschließlich im Wege der Vollharmonisierung oder die Verpflichtung der Mitgliedstaaten zum „copy-and-paste“ und damit zum geradezu sklavischen Nachvollzug unionsrechtlicher Vorgaben trotz an sich gegebener mitgliedstaatlicher Gestaltungsspielräume im Falle einer Mindestharmonisierung ist unvereinbar mit dem Grundsatz, wonach die Mitgliedstaaten soweit möglich ihre Zuständigkeiten auf lokaler, regionaler oder zentraler Ebene wahrnehmen. ${ }^{77}$ Die Kompetenz zur Kontrolle der Einhaltung des Subsidiaritätsprinzips gegenüber Rechtsetzungsinitiativen der Kommission wurde mit dem Vertrag von Lissabon den nationalen Parlamenten bzw. deren Kammern eingeräumt. ${ }^{78}$

Und nach dem in Art. 5 Abs. 4 EUV definierten Prinzip der Verhältnismäßigkeit „gehen die Maßnahmen der Union inhaltlich wie formal nicht über das zur Erreichung der Ziele der Verträge erforderliche Maß hinaus“. Insb. erwächst aus dem Verhältnismäßigkeitsprinzip eine Pflicht der Union, bei mehreren gleich geeigneten Mitteln das gelindeste zu wählen (z.B. Richtlinie vor Verordnung; Mindestvor Vollharmonisierung; Rahmenregelungen vor Detailregelungen). ${ }^{79}$ Aus diesem Grund verweisen etwa in der Interinstitutionellen Vereinbarung zur besseren Rechtsetzung aus 2003 Parlament, Rat und Kommission ,,auf die Definition der Richtlinie [Art. 288 AEUV] sowie auf die einschlägigen Bestimmungen des Protokolls über die Anwendung der Grundsätze der Subsidiarität und der Verhältnismäßigkeit. Bei ihren Richtlinienvorschlägen achtet die Kommission auf eine angemessene Ausgewogenheit zwischen den allgemeinen Grundsätzen und den detaillierten Bestimmungen, um einen übermäßigen Rückgriff auf gemeinschaftliche Durchführungsmaßnahmen zu vermeiden." 80

77 Wie hier W. Schroeder (Fn. 18), S. 5; ders. (Fn. 19), JRP 2018, S. 227, 233; M. Payrhuber/U. Stelkens (Fn. 10), S. 213.

78 Vgl. Art. 6 und Art. 7 Protokoll über die Anwendung der Grundsätze der Subsidiarität und der VerhältnismäBigkeit (Prot. Nr. 2 zum Vertrag von Lissabon).

79 Vgl. im Detail F. Leidenmühler, Europarecht, 3. Aufl. 2017, S. 38.

80 Interinstitutionelle Vereinbarung „Bessere Rechtsetzung“(AB1. EU 2003 C 321/1, P 13). 
c) Charakter der Rechtssatzform Richtlinie gem. Art. 288 Abs. 3 AEUV

Art. 288 AEUV, der die Rechtssatzformen des sekundären Unionsrechts definiert, sieht für die Richtlinie im Gegensatz zur Verordnung, die „,in allen ihren Teilen verbindlich“ ist (Abs. 2 leg. cit.), vor, dass Richtlinien ,hinsichtlich des zu erreichenden Ziels verbindlich [sind], jedoch den innerstaatlichen Stellen die Wahl der Form und der Mittel [überlassen]“" (Art. 288 Abs. 3 leg. cit.). Die Rechtssatzform Richtlinie ist daher idealtypischerweise darauf angelegt, den Mitgliedstaaten bei der Umsetzung nationale Spielräume zu belassen, u.U. auch für Standarderhöhungen. ${ }^{81}$ Die Konstruktion der Richtlinie ist somit ein Kompromiss zwischen der weitest möglichen Bewahrung nationaler Eigenheiten - die verfassungsrechtliche, ganz profane materielle oder auch lediglich politische Gründe haben können - einerseits und den Erfordernissen eines EU-weit einheitlichen Rechts andererseits. $^{82}$

Würde eine Richtlinie mit sehr hoher Regelungsdichte keinerlei Umsetzungsspielraum belassen, so käme sie in ihren Wirkungen einer Verordnung gleich, ${ }^{83}$ was einen Formenmissbrauch vor allem dort darstellen würde, wo dem Unionsgesetzgeber nicht die Wahl zwischen mehreren „Maßnahmen“ überlassen ist, sondern er auf die Rechtssatzform der Richtlinie festgelegt ist. Durch eine bis ins Detail determinierte Vollharmonisierungs-Richtlinie wird nicht nur eine Unter-, sondern auch eine Obergrenze des regulierten Bereichs gezogen. Genau gesehen gibt es eigentlich dann auch keinen Zwischenbereich, und die Freiheit der Mitgliedstaaten beschränkt sich auf eine Einpassung in den nationalen Kontext i.S.e. „,copyand-paste" einschließlich der Beibehaltung rechtlicher Begriffe. Durch solch einen Befehl zu sklavischem Nachvollzug, bei dem jede Abweichung, die sich im Ergebnis in irgendeiner Weise niederschlägt, einen Umsetzungsfehler bedeuten würde, ${ }^{84}$ wäre den Mitgliedstaaten jeder im Falle der Richtlinie definitionsgemäß vorhandene Spielraum verbaut. ${ }^{85}$

\section{d) Primärrechtliche Kompetenzschranken}

Für einzelne Sachkompetenzen sind mitgliedstaatliche Spielräume primärrechtlich garantiert und können somit vom Unionsgesetzgeber gar nicht einer Vollharmonisierung unterworfen werden. Dies betrifft insb. den Bereich der Sozialpolitik und dabei z.B. die Verbesserung der Arbeitsumwelt zum Schutz der Gesundheit und Sicherheit der Arbeitnehmer, die Arbeitsbedingungen, die soziale Sicherheit

81 Vgl. EWSA, Implementation of better legislation - Effect of the Stoiber Report, 2016, S. 28.

82 Vgl. R. Wieland, „Brüsseler Regelungswut“? - Facetten einer politischen Schimäre, in: Sander/Vetter (Hrsg.), Regelungswut in der EU - Wahrheit oder Mythos?, 2007, S. 9, 20; T. Jäger (Fn. 18), S. 38.

83 Vgl. dazu J. Köndgen, Die Rechtsquellen des Europäischen Privatrechts, in: Riesenhuber (Hrsg.), Europäische Methodenlehre, 3. Aufl. 2015, S. 95, 105.

84 Vgl. G. Schulze, Ökonomik der Vollharmonisierung im Gemeinschaftsprivatrecht, in: Gsell/Herresthal (Hrsg.), Vollharmonisierung im Privatrecht, 2009, S. 63, 77.

85 Wie hier vgl. W. Schroeder (Fn. 19), JRP 2018, S. 227, 232. 
und den sozialen Schutz der Arbeitnehmer oder die Vertretung der Arbeitnehmerinteressen und die Mitbestimmung (Art. 153 Abs. 1 lit. a-i AEUV). Nach Art. 153 Abs. 2 lit. b AEUV dürfen auf diesen Gebieten durch Richtlinien lediglich Mindestvorschriften erlassen werden. ${ }^{86}$ Hier sind die Mitgliedstaaten zudem im Falle eines bestehenden höheren Standards sogar unionsrechtlich zur Aufrechterhaltung dieses über die Richtlinien-Vorgaben hinausgehenden Schutzniveaus verpflichtet. Die strikte Ablehnung von sog. „Gold Plating“ würde dem hier verankerten vertraglichen Charakter europäischer Mindeststandards widersprechen.

\section{e) Primärrechtliche Schutzverstärkungsklauseln}

Primärrechtliche Schutzverstärkungsklauseln (wie etwa Art. 114 Abs. 4 und 5 AEUV für die Binnenmarktharmonisierung oder Art. 193 AEUV für den Umweltschutz) garantieren den Mitgliedstaaten die Möglichkeit der Aufrechterhaltung mit den Verträgen vereinbarer höherer einzelstaatlicher Standards unter den jeweils vorgesehenen Voraussetzungen, zum Teil auch erst nach Genehmigung durch die Kommission.

So sind im Bereich der Binnenmarktharmonisierung (Art. 114 AEUV), die auch den gesamten Bereich der binnenmarktbezogenen Verbraucherschutzmaßnahmen umfasst, ${ }^{87}$ zwar Vollharmonisierungen zulässig, aufgrund der Schutzverstärkungsklauseln der Abs. 4 und 5 leg. cit. sind aber dennoch einzelstaatliche Abweichungen zugunsten höherer Standards weiterhin - unter Kontrolle der Kommission (Abs. 6 leg. cit.) - möglich. ${ }^{88}$ Auch im Umweltbereich ist es den Mitgliedstaaten gem. Art. 193 AEUV jedenfalls gestattet, ,verstärkte Schutzmaßnahmen beizubehalten oder zu ergreifen“, sodass hier Vollharmonisierungen von vornherein ausgeschlossen sind.

Es zeigt sich damit, dass zum einen in gewissen Bereichen lediglich Mindestharmonisierungen auf Unionsebene zulässig sind und in anderen Bereichen trotz Vollharmonisierung weiterhin mitgliedstaatliche Spielräume für Schutzstandarderhöhungen bestehen. ${ }^{89}$

86 Dementsprechend heißt es etwa in den Erwägungsgründen der Rahmen-Richtlinie über Sicherheit und Gesundheitsschutz bei der Arbeit (RL 89/391/EWG des Rates vom 12.6.1989, ABl. EG 1989 L 183/1): „Durch diese Richtlinie kann keine mögliche Einschränkung des bereits in den einzelnen Mitgliedstaaten erzielten Schutzes gerechtfertigt werden; die Mitgliedstaaten haben sich gemäß dem Vertrag verpflichtet, die bestehenden Bedingungen in diesem Bereich zu verbessern und sich eine Harmonisierung bei gleichzeitigem Fortschritt zum Ziel gesetzt.“ [...] „Es ist Aufgabe der Mitgliedstaaten, in ihrem Gebiet die Sicherheit und den Gesundheitsschutz von Arbeitnehmern zu verbessern.“ [...] „Die Rechtsvorschriften der Mitgliedstaaten auf dem Gebiet der Sicherheit und des Gesundheitsschutzes am Arbeitsplatz sind sehr unterschiedlich und sollten verbessert werden. Die einschlägigen einzelstaatlichen Bestimmungen, die weitergehend durch technische Vorschriften bzw. freiwillig eingeführte Normen ergänzt werden, können zu einem unterschiedlichen Grad der Sicherheit des Gesundheitsschutzes führen und eine Konkurrenz entstehen lassen, die zu Lasten der Sicherheit und des Gesundheitsschutzes geht.

87 Vgl. Art. 169 Abs. 2 lit. a AEUV.

88 Sog. „Gold Plating“ ist mithin auch im Falle einer Vollharmonisierung möglich, es sind lediglich die erschwerten Voraussetzungen im Wege der primärrechtlichen Schutzverstärkungsklauseln zu beachten.

89 Vgl. W. Schroeder (Fn. 19), JRP 2018, S. 227, 232. 


\section{f) Grundentscheidung der Verträge zugunsten hoher Schutzstandards}

Die vorangehend dargestellten sowie viele weitere primärrechtliche Bestimmungen (z.B. Art. 9, Art. 11, Art. 12, Art. 168 Abs. 1, Art. 169 Abs. 1 oder Art. 191 Abs. 2 AEUV) zeigen eine Grundentscheidung der Verträge zur Schaffung und Erhaltung hoher Schutzstandards in den Bereichen Umwelt, Gesundheit und Verbraucherschutz. ${ }^{90}$ Diese bindet sowohl die Kommission als auch den Unionsgesetzgeber, auch wenn sie über ein gewisses Ermessen verfügen, welchen Standard sie als angemessen erachten. ${ }^{91}$ Ein gänzliches Verunmöglichen höherer mitgliedstaatlicher Standards wäre damit aber jedenfalls nicht vereinbar. Damit zeigt sich zudem auch die Widersprüchlichkeit der Ablehnung des sog. „Gold Plating“: Während zahlreichen Bestimmungen des Primärrechts eine Grundentscheidung zugunsten hoher Schutzniveaus zu entnehmen ist, die eine Abwärtsspirale bei den Standards verhindern soll, wird eine solche durch 1:1-Umsetzungen sekundärrechtlicher Minimalvorgaben durch die Mitgliedstaaten gerade betrieben.

\section{Ein rechtspolitisches Fazit}

Die Option der Mitgliedstaaten, Richtlinien inhaltlich überzuerfüllen, etwa indem in den Bereichen Umwelt-, Verbraucher- oder Arbeitnehmerschutz höhere nationale Standards aufrechterhalten oder neu eingeführt werden, ist primärrechtlich gesichert.

Es liegt aber am jeweiligen Mitgliedstaat, ob er diese Möglichkeit in Anspruch nimmt. Zum einen, indem er schon als Akteur im Rechtserzeugungsprozess auf Ebene der Union (an dem er ja im Rat mitwirkt ${ }^{92}$ ) darauf hinwirkt, dass schon im jeweiligen Sekundärrechtsakt auch entsprechende immanente Abweichungsmöglichkeiten eingeräumt werden (z.B. durch die Vornahme von Mindestharmonisierungen).

Zum anderen, indem er sich nicht durch selbst auferlegte „Gold-Plating“-Verbote bzw. -Rücknahmen ${ }^{93}$ freiwillig der Möglichkeit begibt, die mannigfach bestehenden primärrechtlichen Schutzerhöhungsklauseln auch in Anspruch zu nehmen, um im Falle von Minimalkompromissen ${ }^{94}$ auf Unionsebene seine tradierten höheren nationalen Systeme zur Stärkung des Schutzes benachteiligter oder abhängi-

90 Die Verträge sind eben „keine liberale Charta [...], die eine Harmonisierung auf dem niedrigsten Niveau oder auch nur auf irgendeinem Durchschnitt der früheren mitgliedstaatlichen Niveaus zur Folge hätte" (GA Fennelly, Schlussanträge v. 15.6.2000 in der Rs. C-376/98 [Deutschland/Parlament und Rat], Rn. 85). Vgl. dazu W. Schroeder (Fn. 14), wbl. 2016, S. 361, 365; ders. (Fn. 18), S. 5; ders. (Fn. 19), JRP 2018, S. 227, 234.

91 Vgl. EuGH, Rs. C-58/08 (Vodafone), ECLI:EU:C:2010:321, Slg. 2010, I-4999, Rn. 55, 58, 65.

92 Zum ordentlichen Gesetzgebungsverfahren siehe Art. 289 und Art. 294 AEUV.

93 Vgl. dahingehend für Österreich den Ministerialentwurf einer Sammelnovelle Gold-Plating, 100/ME 26. GP.

94 Vgl. nur S. Wolf, Ein Vorschlag zur Beseitigung von Repräsentations- und Legitimationsdefiziten in Rat und Europäischem Parlament. Überlegungen zur Debatte über die institutionelle Reform der EU, Politische Vierteljahresschrift 2000, S. 730, der darauf hinweist, dass sich oftmals „,der Rat trotz vertraglicher Verpflichtung zu einem qualifizierten Mehrheitsbeschluss nicht zu einem solchen durchringt, sondern entweder Entscheidungsfindungsprozesse solange verschleppt, bis es zu einem Minimalkonsens kommt, oder ,Nichtentscheidungen" trifft" (S. 731 f.). Dahingehend auch S. Immerfall/H. Schöne, Gurkenverordnung und Europamüdig- 
ger Personengruppen oder der Umwelt aufrechtzuerhalten. Eine Selbstverpflichtung zum „,copy-and-paste“ im Falle von Harmonisierungs-Richtlinien ist primärrechtlich weder gefordert noch intendiert und nimmt einem Mitgliedstaat jeglichen Handlungs- und Gestaltungsspielraum. Warum auf diese Eigenständigkeit freiwillig verzichtet werden soll, bleibt gerade vor dem Hintergrund des Subsidiaritätsprinzips rätselhaft. 